\title{
Dual-Band Reconfigurable Antenna With a Very Wide Tunability Range
}

\author{
Nader Behdad, Student Member, IEEE, and Kamal Sarabandi, Fellow, IEEE
}

\begin{abstract}
A new technique for designing dual-band reconfigurable slot antennas is presented. Dual-frequency operation is achieved by loading a slot antenna with two lumped variable capacitors (varactors) placed in proper locations along the slot. Loading the slot antenna with lumped capacitors shifts down the resonant frequencies of the first and second resonances of the antenna. However, these frequency shifts depend not only on the values of the capacitors, but also on their locations along the slot antenna. Here, it is shown that by choosing the locations of the varactors appropriately, it is possible to obtain a dual-band antenna whose first and second resonant frequencies can be controlled individually. In other words, the frequency of either the first or the second band can be fixed, while the other one is electronically tuned. Using such a design, an electronically tunable dual-band antenna is designed and fabricated using two identical varactors having a capacitance range of $0.5-2.25 \mathrm{pF}$. The antenna is shown to have a frequency ratio $\left(f_{R}=f_{2} / f_{1}\right)$ ranging from 1.3 to 2.67. An important feature of this antenna is its consistent radiation pattern, polarization, and polarization purity at both bands and across its entire tunable frequency range.
\end{abstract}

Index Terms-Multifrequency antennas, multiple band antennas, reconfigurable antennas, slot antennas.

\section{INTRODUCTION}

A VAILABILITY of different unlicensed frequency bands in the congested spectrum of electromagnetic (EM) waves, compatibility of wireless devices with different standards (i.e, GSM and CDMA), and interference reduction in a two-way radio system are typical reasons why many existing wireless systems make use of two different simultaneous frequency bands. Whatever the reason may be, this undoubtedly will add to the complexity of these systems. In particular, the RF circuitry and antennas must be designed to be able to operate at multiple frequency bands. Recent advancements in antenna technology, the availability of efficient computer-aided design (CAD) tools, and the availability of fast and powerful computers have resulted in a variety of different techniques for designing low-profile, cost effective, and highly efficient multiple-frequency antennas [1]-[9]. Many of the techniques used for designing dual-band antennas make use of certain approaches to manipulate the current distribution of one of the higher order resonant modes of the structure to change its resonant frequency as well as current distribution. For example,

Manuscript received June 7, 2005; revised September 9, 2005. This work was supported in part by the Engineering Research Centers program of the National Science Foundation under NSF award Number EEC-9986866.

The authors are with The Radiation Laboratory, Department of Electrical Engineering and Computer Science, The University of Michigan, Ann Arbor, MI 48109 USA (e-mail: behdad@umich.edu).

Digital Object Identifier 10.1109/TAP.2005.863412 in [2], a rectangular patch antenna is modified by a slot insertion at a particular location in order to alter one resonant mode more than the other and achieve a dual-band operation. However, the range of achievable frequency ratios $\left(f_{R}=f_{2} / f_{1}\right.$, the ratio of the frequency of operation of the second band $f_{2}$, to that of the first one $f_{1}$ ) for this technique is limited to $1.6 \leq f_{R} \leq 2$. This idea was later applied to circular and triangular patch antennas where frequency ratios of $1.3 \leq f_{R} \leq 1.4$ and $1.35 \leq f_{R} \leq 1.5$ were, respectively, achieved [3], [4]. Variations of these techniques with differently shaped slots and patches have also been investigated and discussed in detail in [1, Ch. 4].

Slot antennas belong to another category of low-profile printed antennas that show higher efficiency and can provide multiband operation. At the same time, the configuration and radiation characteristics of slot antennas appear to be more amenable to miniaturization and reconfigurability [10]-[12]. In particular, the uniplanar nature of these antennas allows for simple integration of active or passive lumped components into the topology of the antenna without the need for having via holes. Examples of different techniques that are used to design dual-band slot antennas are presented in [5]-[9]. In [5], a single-element dual-band CPW-fed slot antenna with similar radiation patterns at both bands is studied. In [6], a compact dual-band CPW-fed slot antenna, with a size reduction of about $60 \%$ compared to a conventional slot antenna, is studied. Other topologies have also been used to achieve dual- or multiband operations. Examples of these include structures that make use of parasitic elements [7], multiple radiating elements [8], or create fictitious resonant modes [9]. These approaches, however, can only be used to design antennas that have fixed dual- or multiband characteristics.

Electronic reconfigurability is usually achieved by incorporating switches, variable capacitors, phase shifters, or ferrite materials in the topology of the antenna [12]-[16]. Most frequently, lumped components such as PIN diodes, varactor diodes, or MEMS switches or varactors are used in the design of reconfigurable antennas. These components may be used to electronically change the frequency response (e.g., see [12]-[14], and [15]), radiation patterns (e.g., see [16]), gain, or a combination of different radiation parameters of such antennas. Recently, a dual-band slot antenna was proposed that uses a single varactor to achieve tunability in only its second band of operation and has a fixed first band. The frequency ratio of this antenna can electronically be tuned from 1.2 to 1.65 (or equivalently $30 \%$ tunability) [17]. In this paper, we use a similar approach to design a far more versatile dual-band antenna with a greatly enhanced tunability range. It is shown that by strategically placing 
two varactor diodes along a bent slot antenna, an agile dual-band tunable antenna can be achieved. In this design, the frequency of one of the bands (either $f_{1}$ or $f_{2}$ ) can be fixed at will and the frequency of the other band can continuously be tuned over a wide frequency range. Furthermore, if desired, the frequencies of both bands can simultaneously be tuned over a wide frequency range by changing the bias voltages of the varactors. Measurement results indicate that frequency ratios in the range of $1.3 \leq f_{R} \leq 2.67$ (more than one octave tunability) can be achieved. The antenna uses a simple feed structure and matching network to achieve excellent simultaneous impedance match at both frequencies of operation and over its entire tuning range. Furthermore, the antenna shows similar radiation patterns and polarization at both bands with low levels of cross-polarized radiation. Most importantly the radiation patterns of the antenna remain practically unchanged as its frequency response is tuned over the wide tunable frequency range of operation.

\section{LOADED SLOT ANTENNAS FOR DUAL-BAND OPERATION}

\section{A. Resonant Frequencies of a Loaded Slot}

A narrow slot antenna, at its first resonance, may be considered as a $\lambda / 2$ transmission line, short circuited at both ends [18]. Loading such a structure with two capacitors as shown in Fig. 1, increases the line capacitance at two points and, therefore, reduces the frequencies of its first- and all higher-order resonances. This reduction, however, is not uniform and depends on the locations of the capacitors, their values, and the slot line impedance. The transmission line equivalent circuit model shown in Fig. 1 can be used to determine the resonant frequencies of this loaded slot antenna. Transverse resonant condition [19] requires that

$$
Z_{R}+Z_{L}=0
$$

where $Z_{R}$ and $Z_{L}$ are the input impedances to the right and left of the reference point, as shown in Fig. 1 . In this case, $Z_{R}$ and $Z_{L}$ can simply be obtained from the following equations

$$
\begin{aligned}
Z_{R} & =\frac{\jmath Z_{0} \tan \left(\theta-\theta_{2}\right)}{1-\omega C_{2} Z_{0} \tan \left(\theta-\theta_{2}\right)} \\
Z_{L} & =\jmath Z_{0} \frac{\tan \left(\theta_{1}\right)+\tan \left(\theta_{2}-\theta_{1}\right)\left[1-\omega C_{1} Z_{0} \tan \left(\theta_{1}\right)\right]}{1-\omega C_{1} Z_{0} \tan \left(\theta_{1}\right)-\tan \left(\theta_{1}\right) \tan \left(\theta_{2}-\theta_{1}\right)} \\
\theta & =\beta(\omega) \ell, \quad \theta_{1}=\beta(\omega) \ell_{1}, \quad \theta_{2}=\beta(\omega) \ell_{2}
\end{aligned}
$$

where $\beta(\omega)$ is the frequency-dependent propagation constant of the slotline. In addition to being a function of frequency, $\beta$ is a function of the slotline width, relative dielectric constant $\left(\epsilon_{r}\right)$, and thickness $(h)$ of the substrate that the slotline is printed on, if any. $\beta(\omega)$ can easily be calculated from the expressions given in [20] and [21]. Using (2) and (3) in (1) an expression for the resonant condition of the loaded transmission line of Fig. 1 can be achieved

$$
\begin{aligned}
& \left\{\tan \left(\theta_{1}\right)+\tan \left(\theta_{2}\right)\left[1-\omega C_{1} Z_{0} \tan \left(\theta_{1}\right)\right]\right\} \\
& \quad \cdot\left(1-\omega C_{2} Z_{0} \tan \left(\theta-\theta_{2}\right)\right) \\
& \quad+\left\{1-\omega C_{1} Z_{0} \tan \left(\theta_{1}\right)-\tan \left(\theta_{2}\right) \tan \left(\theta_{1}\right)\right\} \\
& \quad \cdot \tan \left(\theta-\theta_{2}\right)=0 .
\end{aligned}
$$

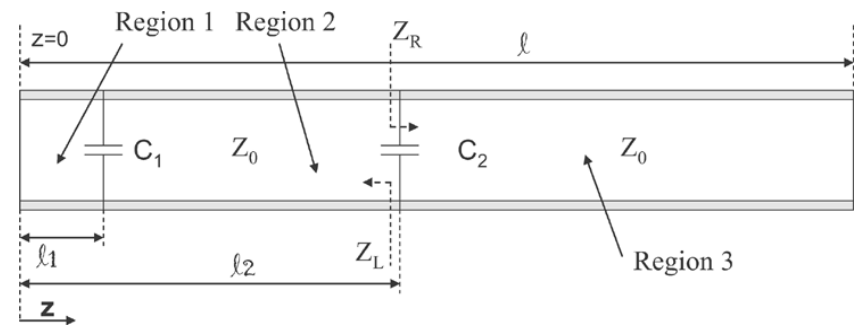

Fig. 1. Transmission line model of a slot antenna loaded with two lumped capacitors.

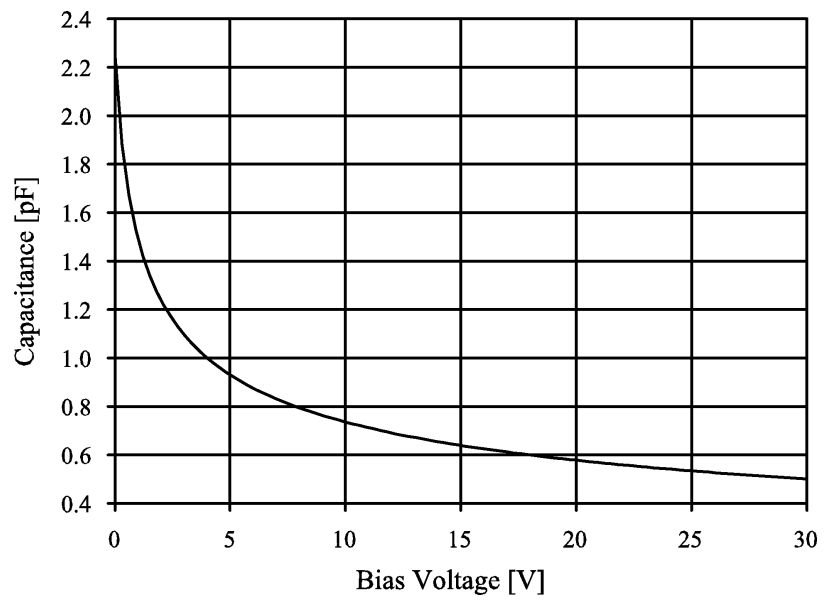

Fig. 2. Typical capacitance of a SMTD3001 varactor (from Metelics Corp.) as a function of its bias voltage.

The roots of this expression are the resonant frequencies of the loaded slot antenna shown in Fig. 1. As is observed from (5), these resonant frequencies have explicit dependence to the variables $\ell_{1}, \ell_{2}, C_{1}$, and $C_{2}$. Therefore, by fixing one or more of these variables and changing the rest, the resonant frequencies of the slot antenna can be changed. In particular, if $\ell_{1}$ and $\ell_{2}$ are fixed, $C_{1}$ and $C_{2}$ can be tuned electronically (by using varactor diodes) to achieve a dual-band reconfigurable antenna. Here $C_{1}$ and $C_{2}$ are replaced by two identical SMTD3001 varactors from Metelics Corp. The varactor's capacitances varies from 2.25 to $0.5 \mathrm{pF}$ as the bias voltage is increased from 0 to $30 \mathrm{~V}$ (see Fig. 2).

In this paper, our goal is to maximize the tunability range of the antenna's frequency ratio (i.e., to maximize the tunability range of $f_{R}=f_{2} / f_{1}$ ) for a given set of varactors. In order to do that, (5) is solved for fixed values of $\ell_{1}$ and $\ell_{2}$ and different capacitor values in the range of $0.5 \mathrm{pF} \leq C_{1}, C_{2} \leq 2.25 \mathrm{pF}$ to obtain $f_{1}\left(C_{1}, C_{2}\right)$ and $f_{2}\left(C_{1}, C_{2}\right)$. Once these values are obtained, $f_{R}\left(C_{1}, C_{2}\right)=f_{2}\left(C_{1}, C_{2}\right) / f_{1}\left(C_{1}, C_{2}\right)$ is calculated. In order to quantify the tunability range of $f_{R}$, for fixed varactor locations, the parameter $\alpha\left(\ell_{1}, \ell_{2}\right)$ is defined as follows:

$$
\alpha\left(\ell_{1}, \ell_{2}\right)=\frac{\max \left\{f_{R}\left(C_{1}, C_{2}\right)\right\}}{\min \left\{f_{R}\left(C_{1}, C_{2}\right)\right\}} \text {, for fixed } \ell_{1} \text { and } \ell_{2} \text {. }
$$

This procedure is then repeated for different values of $\ell_{1}$ and $\ell_{2}$ to obtain $\alpha\left(\ell_{1}, \ell_{2}\right)$ for a range of $\ell_{1}$ and $\ell_{2}$ values. Once this function is obtained, the varactor locations along the slot, $\ell_{1}$ and $\ell_{2}$, are chosen to maximize $\alpha$ and consequently maximize the tunability range of $f_{R}$. This procedure is performed for a 


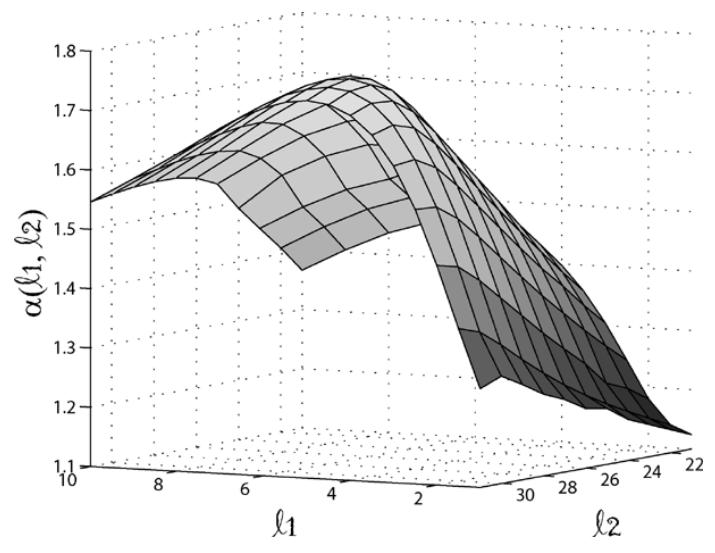

Fig. 3. Calculated values of $\alpha=\max \left\{f_{R}\left(C_{1}, C_{2}\right)\right\} / \min \left\{f_{R}\left(C_{1}, C_{2}\right)\right\}$ for $0.5 \mathrm{pF} \leq C_{1}, C_{2} \leq 2.25 \mathrm{pF}$ and different values of $\ell_{1}$ and $\ell_{2}$. Here $f_{R}=f_{2} / f_{1}$ for a dual-band slot antenna loaded with two varactors located at $\ell_{1}$ and $\ell_{2}$ from one end.

straight slot antenna with a length of $\ell=62 \mathrm{~mm}$ and width of $w=1 \mathrm{~mm}$ printed on a 0.5 -mm-thick substrate with dielectric constant of $\epsilon_{R}=3.4$. The slotline characteristics impedance and frequency-dependent propagation constant $\left(\beta(\omega)\right.$ and $Z_{0}$ in (4) and(5)) are calculated from the formulae given in [20] and [21]. Using the procedure mentioned in the preceding paragraph, $\alpha\left(\ell_{1}, \ell_{2}\right)$ is calculated for this particular slot antenna and is presented in Fig. 3. It is observed that $\alpha$ clearly has a maximum that occurs for $\ell_{1}=4.5 \mathrm{~mm}$ and $\ell_{2}=29 \mathrm{~mm}$.

It should be noted that solutions of (5) are only approximations of the real resonant frequencies of the actual antenna, since the effects of radiation from the slot antenna and more importantly the effects of the feed network parameters on the resonant frequency of the antenna are ignored. A more accurate method of predicting the actual resonant frequencies of the structure is to use full wave simulations. In this case, the effects of finite ground planes and dielectric substrates, radiation, and feed network parameters on the antenna's resonant frequencies can be taken into account. This is performed in [17] where both methods are used to predict the resonant frequencies of a similar slot antenna and the results are presented and compared. It is shown that despite the simplicity of the analytical method, the maximum error between its predicted resonant frequencies and those obtained from full-wave simulations remains below $8 \%$.

\section{B. Field Distribution Along the Loaded Slot Antenna}

In addition to having a large tuning range for $f_{R}$, having consistent radiation patterns at both bands and over the entire tuning range is also of significant importance. The radiation patterns of a slot antenna are mainly determined by the electric field (i.e., voltage) distribution over the aperture. For an unloaded slot antenna, the electric field (magnetic current) distribution at the first and second resonance modes resemble that of a half sine wave and a full sine wave, respectively. The symmetric field distribution at the first band results in a radiation pattern with maximum directivity at the antenna's broadside whereas the antisymmetric field distribution of the second band, generates a pattern with a null at broadside. To mitigate this undesired characteristic, it is necessary to study the electric field distribution of the loaded slot antenna. The simple transmission line model of Fig. 1, is

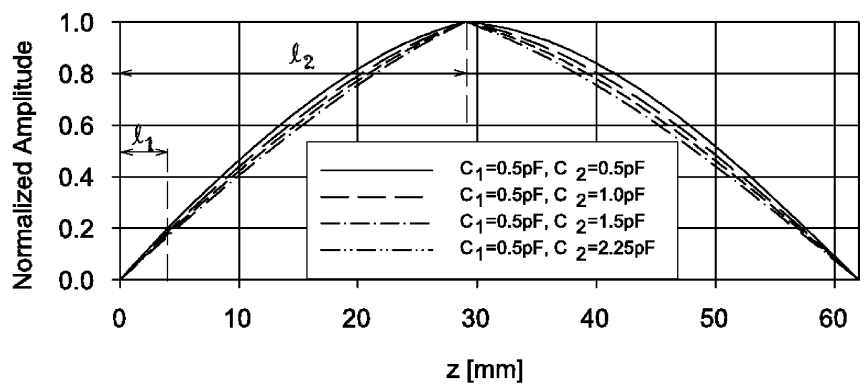

(a)

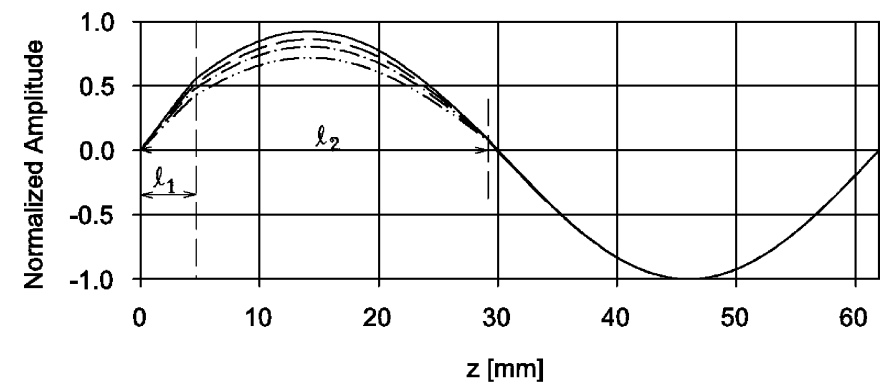

(b)

Fig. 4. Voltage distribution across the slotline shown in Fig. 1 for a fixed value of $C_{1}=0.5 \mathrm{pF}$ and different $C_{2}$ values. (a) First resonance. (b) Second resonance.

sufficient to qualitatively study the field distribution. The voltages and currents in Regions 1, 2, and 3 can be expressed as [19]

$$
\begin{aligned}
& V_{n}(z)=V_{n}^{+} e^{-\jmath \beta z}+V_{n}^{-} e^{\jmath \beta z} \\
& I_{n}(z)=\frac{V_{n}^{+}}{Z_{0}} e^{-\jmath \beta z}-\frac{V_{n}^{-}}{Z_{0}} e^{\jmath \beta z}
\end{aligned}
$$

where the subscript $n$ refers to region 1,2 , or 3 . The boundary conditions at $z=0, \ell_{1}, \ell_{2}$, and $\ell$ require that

$$
\begin{aligned}
V_{1}(0) & =0, V_{1}\left(\ell_{1}\right)=V_{2}\left(\ell_{1}\right) \\
V_{2}\left(\ell_{2}\right) & =V_{3}\left(\ell_{2}\right), V_{3}(\ell)=0 \\
I_{1}\left(\ell_{1}\right) & =I_{2}\left(\ell_{1}\right)+\jmath \omega C_{1} V_{1}\left(\ell_{1}\right) \\
I_{2}\left(\ell_{2}\right) & =I_{3}\left(\ell_{2}\right)+\jmath \omega C_{2} V_{3}\left(\ell_{2}\right) .
\end{aligned}
$$

Simultaneous solution of (7) and (8) subject to the boundary conditions of $(9-a)-(9-d)$, yields the voltage distribution at the first and second resonances of the loaded line. Fig. 4 shows the slot antenna's voltage distribution at its first and second resonances for an antenna with $\ell=62 \mathrm{~mm}, w=1 \mathrm{~mm}$, $\ell_{1}=4.5 \mathrm{~mm}$, and $\ell_{2}=29 \mathrm{~mm}$ printed on a $0.5-\mathrm{mm}$-thick substrate with $\epsilon_{r}=3.4$. In this figure, $C_{1}$ is fixed at $0.5 \mathrm{pF}$ and $\mathrm{C}_{2}$ is varied. Fig. 5 shows the voltage distribution of the two bands for a fixed $C_{2}=0.5 \mathrm{pF}$ and different values of $C_{1}$. It is observed that the field distribution and the resonant frequency of the first mode is mainly affected by changes in $C_{2}$ whereas the field distribution and resonant frequency of the second mode is mainly affected by changes in $C_{1}$. Examining Figs. 4(a) and 5 (a) reveals that changes in the values of the capacitors does not significantly affect the field distribution of the first mode and hence has minimal effect on its radiation pattern. However, this is not the case for the second band. In particular, Figs. 4(b) and 5(b) show that the field distribution at the second resonance, and hence its radiation pattern, change as capacitor values are 


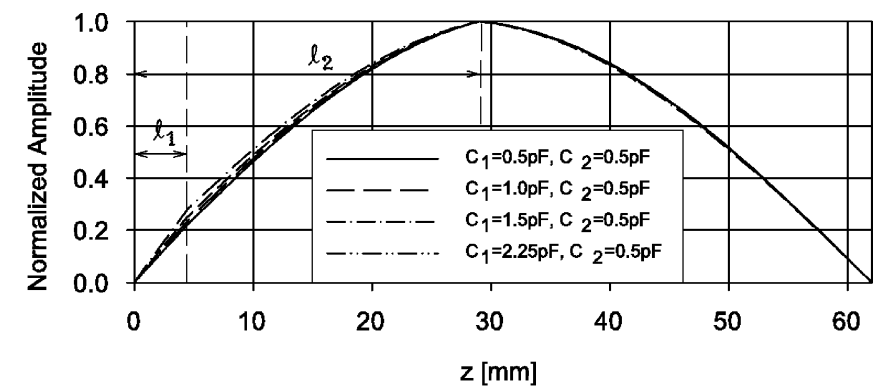

(a)

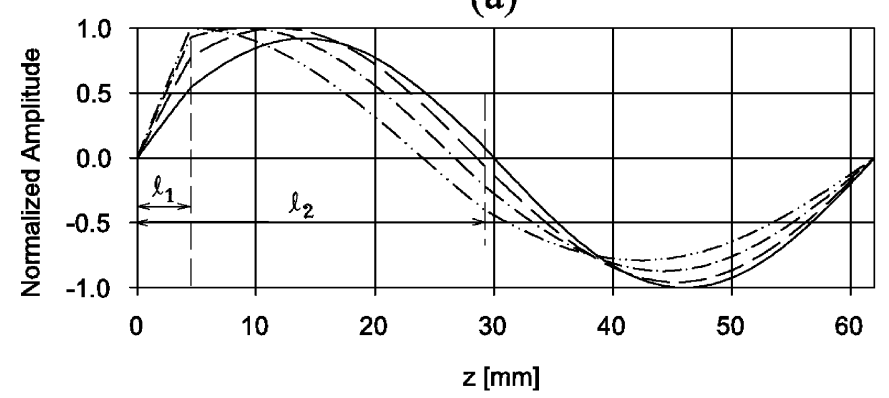

(b)

Fig. 5. Voltage distribution across the slotline shown in Fig. 1 for a fixed $C_{2}=$ $0.5 \mathrm{pF}$ and different $C_{1}$ values. (a) First resonance. (b) Second resonance.

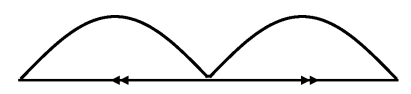

(a)

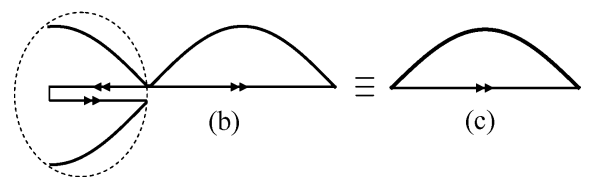

Fig. 6. (a) Simple representation of the magnetic current distribution of a straight slot antenna at its second resonant mode. (b) Magnetic current distribution of the same slot at second resonant mode when it is bent $\lambda_{2} / 4$ away from its edge will roughly be equivalent to (c) that of the first mode of a slot antenna that has half its length.

changed. More importantly, because of its semi anti-symmetric field distribution, this mode will still have a null or a minima at the antenna's broadside.

Fig. 6(a) shows the magnitude and direction of the magnetic current of an unloaded slot antenna at its second resonant mode. The antenna has a length of $\lambda_{2}$, where $\lambda_{2}$ is the slot wavelength at the second resonance. If the antenna is folded at a distance of $\lambda_{2} / 4$ from one end, the magnetic current distribution will assume a form shown in Fig. 6(b). In this arrangement the oppositely directed magnetic currents inside the dashed contour have similar magnitude but opposite direction. Therefore, that part of the antenna does not effectively contribute to the far-field radiated fields and therefore the folded antenna becomes equivalent to the one shown in Fig. 6(c). This way, the radiation pattern of the second mode will be similar to that of the first mode. In addition to solving the radiation pattern problems, this approach reduces the overall length of the antenna by about $25 \%$. However, as a result of the slight antenna miniaturization, the antenna gain and efficiency will slightly be reduced (see [22]). This can be viewed as a tradeoff between having similar radiation patterns at both bands and a smaller antenna with the slight

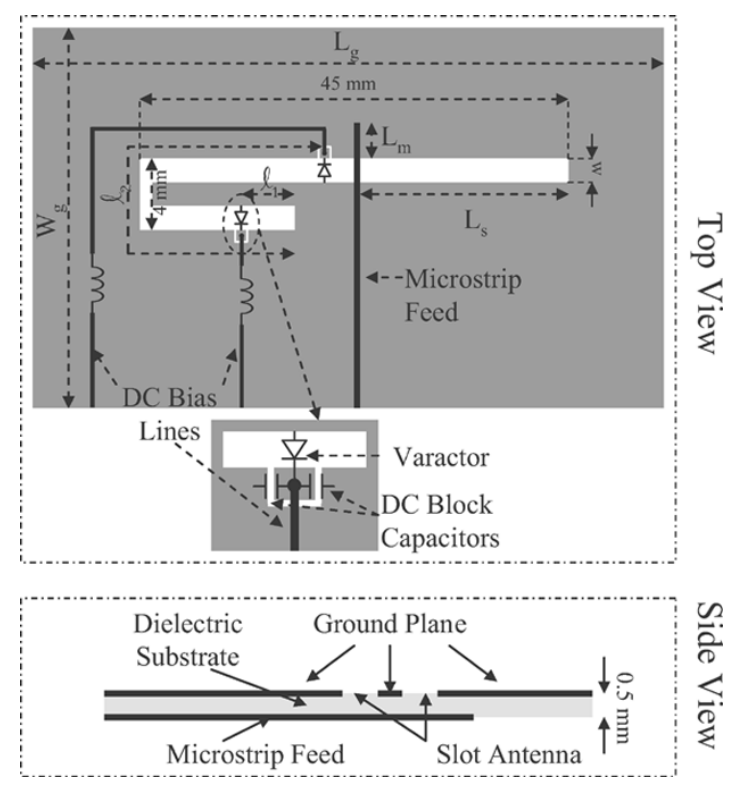

Fig. 7. Schematic of the proposed dual-band bent slot antenna with two varactors.

reduction in antenna efficiency. In the next section, experimental results of such a dual-band reconfigurable antenna are presented and discussed.

\section{EXPERIMENTAL RESULTS}

The schematic of the proposed dual-band slot antenna is shown in Fig. 7. The antenna uses the folded topology described in the previous section with the smaller and larger sections of 15- and 45-mm long, respectively. The overall average length of the antenna is $\ell=62 \mathrm{~mm}$ with $\ell_{1}=4.5 \mathrm{~mm}$ and $\ell_{2}=29 \mathrm{~mm}$. The antenna is designed on a $0.5-\mathrm{mm}$-thick RO4003 substrate with dielectric constant of $\epsilon_{r}=3.4$ and loss tangent of $\tan (\delta)=0.002$ with an overall ground plane size of $15 \mathrm{~cm} \times 11 \mathrm{~cm}$. The antenna is fed with an off-centered open circuited microstrip line with an impedance of $50 \Omega$. Matching is performed by appropriately choosing the location of the microstrip feed and the length of the open circuited line ( $L_{S}$ and $L_{m}$ in Fig. 7) as described in [18] and [23]. Optimum locations of the feed and lengths of the open circuited stubs are determined by performing optimization using a method of moment (MoM) based full-wave simulation software (IE3D) [24]. The antenna is simulated in IE3D and fabricated on the same RO4003 substrate aforementioned. The input reflection coefficient $\left(S_{11}\right)$ of the antenna, for different varactor bias voltages, is measured using a calibrated vector network analyzer (VNA) and the results are presented in Figs. 8 and 9 along with the simulated results. Fig. 8(a) and (b) shows the simulated and measured dual-band responses of the antenna where by applying the appropriate combination of bias voltages $\left(V_{1}\right.$ and $V_{2}$ ) the frequency of the first band is kept fixed and that of the second band is tuned. Similarly, as shown in Fig. 9(a) and (b), it is possible to keep the frequency of the second band stationary and sweep the frequency of the first band. As is observed from these figures, a relatively good agreement between the simulated and measured results are observed. The discrepancies 


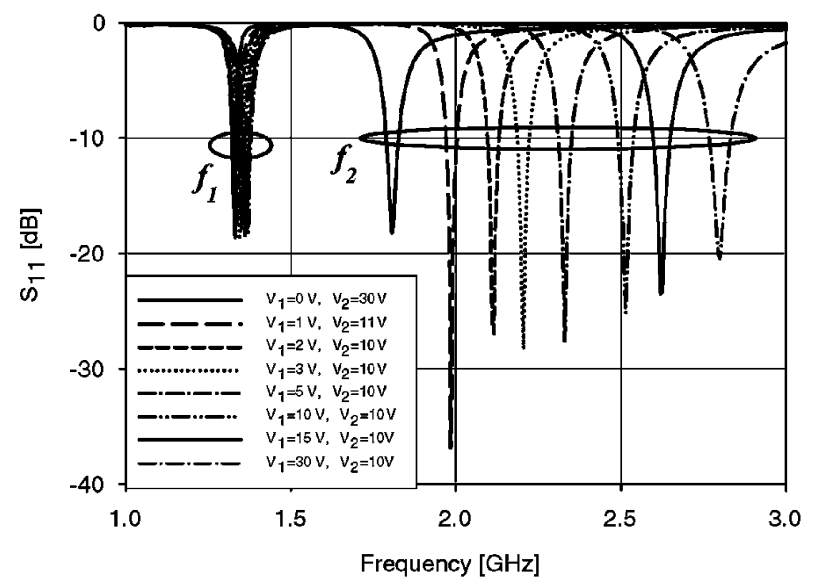

(a)

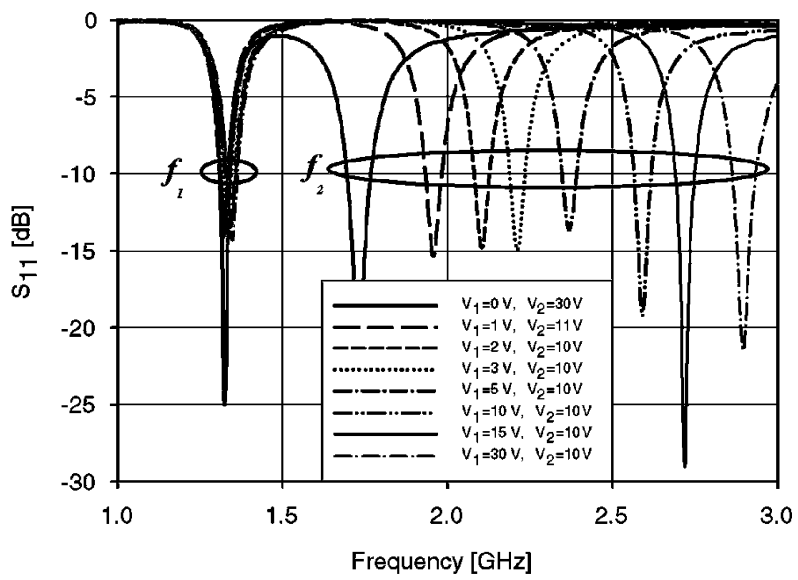

(b)

Fig. 8. (a) Simulated and (b) measured input reflection coefficients of the dual-band tunable antenna of Fig. 7. In this example, the frequency of the first band is kept fixed while that of the second band is varied.

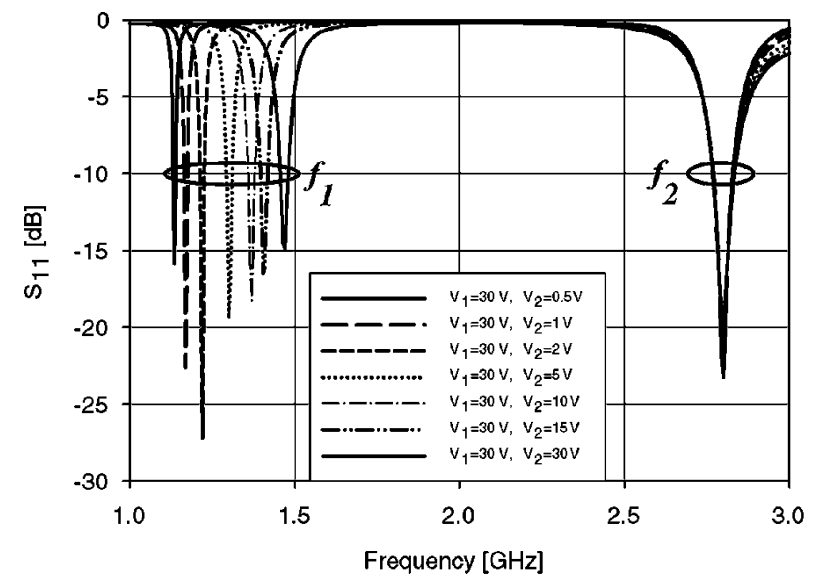

(a)

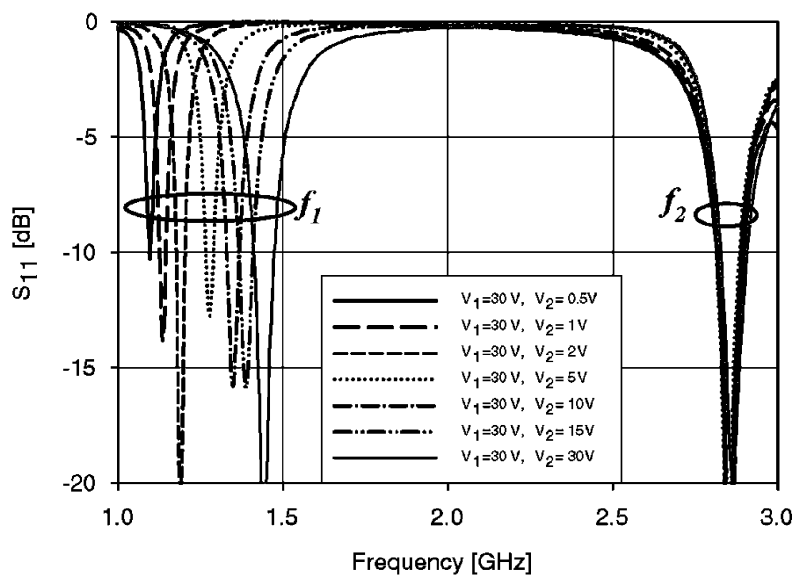

(b)

Fig. 9. (a) Simulated and (b) measured input reflection coefficients of the dual-band tunable antenna of Fig. 7. In this example, the frequency of the second band is kept fixed while that of the first band is varied.

between these results can mostly be attributed to the finiteness of the ground plane and the dielectric substrate (which are considered infinite in the simulations), tolerances in the exact value of the substrate's dielectric constant $( \pm 3 \%$ error specified by manufacturer), alignment errors during the fabrication process, and numerical errors in the software.

The antenna's $S_{11}$ is measured for over 300 different combinations of varactor bias voltages $\left(V_{1}, V_{2}\right)$ and the operation frequencies of the first and second bands are extracted from these measurements and are presented in Fig. 10(a) and (b), respectively. It can be observed that the frequency of the first band is mainly affected by the bias voltage (capacitance) of the second varactor, whereas the frequency of the second band is mostly affected by the capacitance of the first varactor. This is consistent with the slot antenna's electric field distributions shown in Figs. 4 and 5. The reason for this behavior can become clear by considering the electric field distribution of the unloaded slot antenna in the first and second resonant modes. At first resonance, the voltage at the center of the slot is at its maximum whereas it is zero at the second resonance. Therefore, a capacitor that is
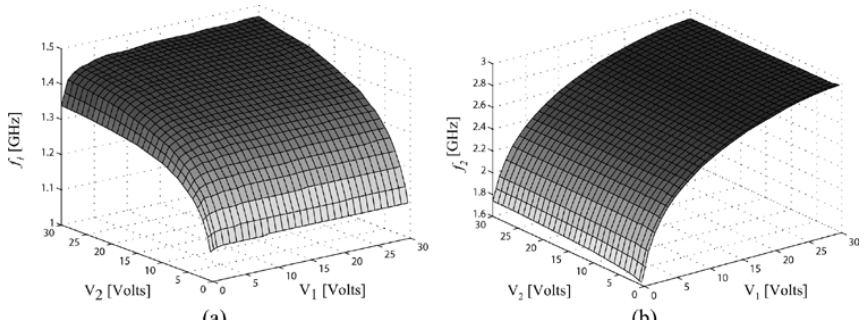

(b)

Fig. 10. Measured frequencies of (a) first band and (b) second band of the tunable dual-band slot antenna shown in Fig. 7 as a function of the varactors' bias voltages. It can be observed that $f_{1}$ is less sensitive to $V_{1}$ and $f_{2}$ is less sensitive to $V_{2}$.

placed at the center of the slot antenna observes a short circuit at the second resonance and does not significantly affect this mode. On the other hand, if a capacitor is placed close to the edge of slot, it observes a much lower impedance at the first resonance than that of the second one and, hence, it mostly affects the second mode. The frequency ratios of the antenna $\left(f_{R}\right)$ as a function of $V_{1}$ and $V_{2}$ are calculated from the measured $S_{11}$ 


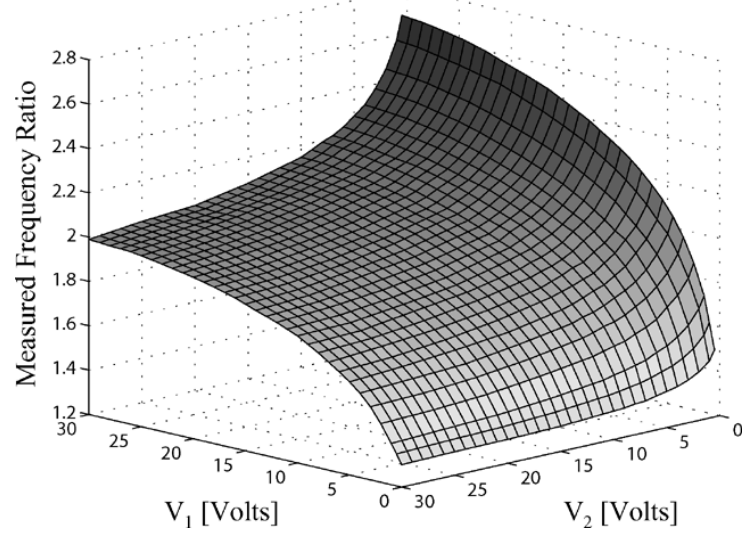

Fig. 11. Measured frequency ratio, $f_{R}=f_{2} / f_{1}$, of the tunable dual-band slot antenna shown in Fig. 7 as a function of the varactors' bias voltages.

TABLE I

PhySicAl PARAMETERS OF THE DUAL-BAND ReCONFIGURABLE SLOT ANTENNA

\begin{tabular}{|c||c|c|c|}
\hline Parameter & $L_{g} \times W_{g}$ & $L_{s}$ & $L_{m}$ \\
\hline Value & $15 \times 11 \mathrm{~cm}^{2}$ & $27.5 \mathrm{~mm}$ & $3.2 \mathrm{~mm}$ \\
\hline Parameter & $\ell$ & $\ell_{1}$ & $\ell_{2}$ \\
\hline Value & $62 \mathrm{~mm}$ & $4.5 \mathrm{~mm}$ & $29 \mathrm{~mm}$ \\
\hline
\end{tabular}

TABLE II

ELECTRICAL PARAMETERS OF THE ANTENNA CORRESPONDING TO LOWEST AND HIGHEST MEASURED $f_{R}$ VALUES

\begin{tabular}{|c|c|c|c|c|}
\hline$\left(V_{1}, V_{2}\right)[$ Volts] & $\left(C_{1}, C_{2}\right)[\mathrm{pF}]$ & $f_{1}[\mathrm{GHz}]$ & $f_{2}[\mathrm{GHz}]$ & $f_{R}$ \\
\hline$(30.0,0.5)$ & $(0.5,1.75)$ & 1.1 & 2.94 & 2.67 \\
\hline$(0.0,30.0)$ & $(2.25,0.5)$ & 1.34 & 1.74 & 1.3 \\
\hline
\end{tabular}

responses and presented in Fig. 11. It is observed that a large range of frequency ratios can be obtained by just changing the varactors' bias voltages. In this case $f_{R}\left(V_{1}, V_{2}\right)$ attains the maximum value of 2.67 and the minimum value of 1.30 as seen from Table II. One of the parameters that affect the resonant frequencies and frequency ratios of this antenna is the dimension of its ground plane. These effects are comprehensively studied in [17], where it is shown that for a fixed dc bias voltage value, decreasing $L_{g}$ and $W_{g}$ reduces the frequency of operation of the first and second bands $\left(f_{1}\right.$ and $\left.f_{2}\right)$ and the frequency ratio of the dual-band antenna. Furthermore, the ground plane size of a slot antenna also affects its gain and radiation patterns as described in [17] and the references therein. The ground plane dimensions, however, are usually determined by the type of application and the constraints on the dimensions of the final product in which the antenna is used. Therefore, the effects of the finite substrate and ground plane size on the performance of the antenna must be taken into account during the design process in order to achieve the desired frequency response and performance.

The co- and cross-polarized radiation patterns of the antenna at the first and second bands are measured for different combinations of bias voltages and are presented in Figs. 12(a)-(d) and 13(a)-(d). Fig. 12(a) and (b) shows the co- and cross-polarized radiation patterns of the antenna, respectively, in its first

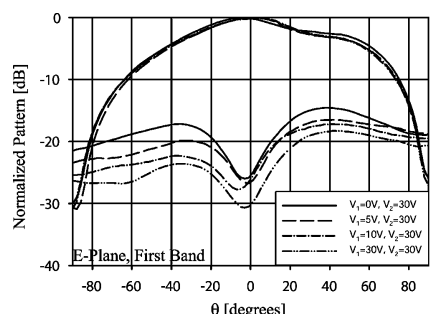

(a)

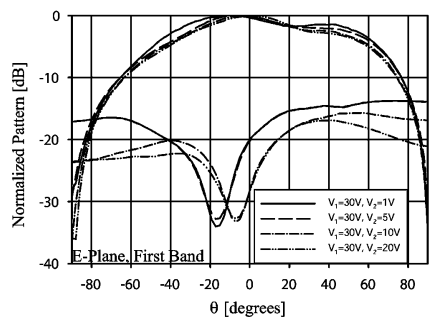

(c)

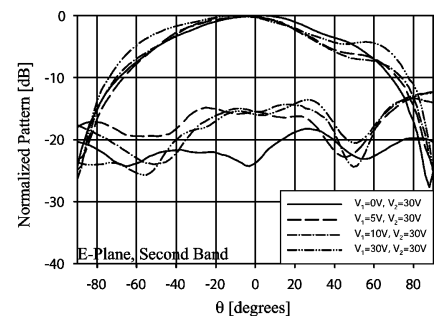

(b)

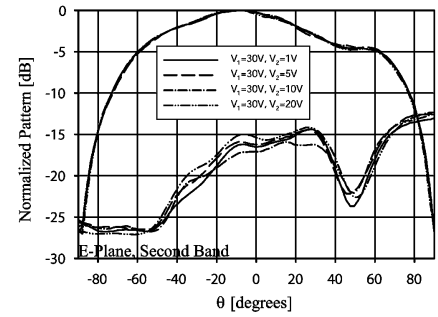

(d)
Fig. 12. Measured E-plane radiation patterns of the tunable dual-band slot antenna shown in Fig. 7 at its first and second bands of operation.

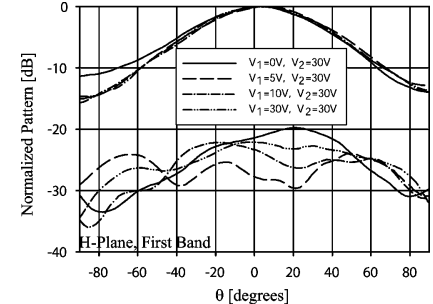

(a)

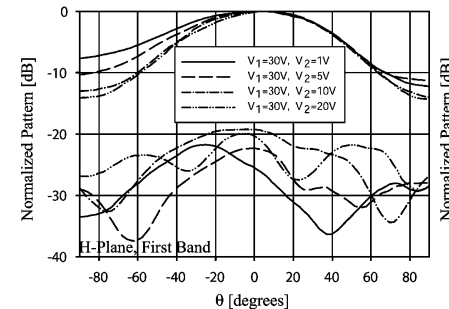

(c)

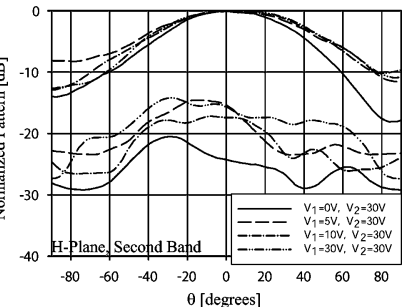

(b)

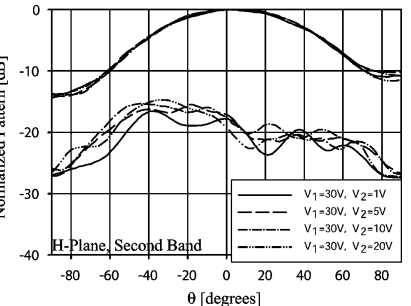

(d)
Fig. 13. Measured H-plane radiation patterns of the tunable dual-band slot antenna shown in Fig. 7 at its first and second bands of operation.

and second bands of operation for a fixed value of $V_{2}$ and variable $V_{1}$ values. It is seen that the radiation patterns at both bands are similar to each other and the antenna has low levels of cross polarized radiation. Furthermore, as expected, changing $V_{1}$ slightly affects the radiation pattern of the second band and has little effect on the patterns of the first band. Nevertheless, for all practical purposes, the radiation patterns remain consistent. Fig. 12(c) and (d) show the same patterns for constant values of $V_{1}$ and variable $V_{2}$ values. In this case, the antenna patterns at the second band remain unaffected whereas the radiation patterns at the first band are slightly affected. In spite of the small changes in the radiation patterns, the antenna has similar radiation patterns at both bands across its entire frequency of operation. Fig. 13(a)-(d) show the H-plane co- and cross-polarized radiation patterns of the antenna for the same varactor bias voltages. Similar to the E-plane patterns, the $\mathrm{H}$-plane patterns of the first and second bands of operation have small levels of 
TABLE III

Measured Gain of the Dual-Band Tunable Antenna at First (Second) Bands of Operation as a Function of Varactor Bias Voltages. All Values ARE in dBi

\begin{tabular}{|c|c|c|c|c|c|}
\hline$V_{1}(\downarrow), V_{2}(\rightarrow)$ & $1 \mathrm{~V}$ & $5 \mathrm{~V}$ & $10 \mathrm{~V}$ & $20 \mathrm{~V}$ & $30 \mathrm{~V}$ \\
\hline $0 \mathrm{~V}$ & $-0.6(-0.4)$ & $0.2(0.1)$ & $0.3(0.2)$ & $0.3(0.2)$ & $0.3(0.2)$ \\
\hline $5 \mathrm{~V}$ & $0.0(1.5)$ & $0.7(1.6)$ & $0.8(1.7)$ & $0.9(1.8)$ & $0.9(1.8)$ \\
\hline $10 \mathrm{~V}$ & $0.2(1.7)$ & $0.8(1.8)$ & $0.9(1.8)$ & $1.1(1.8)$ & $1.1(1.8)$ \\
\hline $20 \mathrm{~V}$ & $0.2(1.7)$ & $1.0(1.8)$ & $1.1(1.8)$ & $1.2(1.8)$ & $1.2(1.8)$ \\
\hline $30 \mathrm{~V}$ & $0.2(1.7)$ & $1.0(1.8)$ & $1.1(1.8)$ & $1.2(1.8)$ & $1.2(1.8)$ \\
\hline
\end{tabular}

cross-polarized radiation and their co-pol components remain unchanged over the entire band of operation. The gain of the antenna, at broadside, is measured using a double-ridged horn antenna as a reference. The measurement results are presented in Table III. It is observed that as the capacitances of the varactors increase (the bias voltages decrease), the antenna gain drops. Increase in capacitance reduces the resonant frequencies of both bands of the antenna and hence, for a fixed physical length, decreases the electrical length of the antenna. This reduction in electrical length (miniaturization) reduces the antenna gain as described in [22]. The antenna efficiency values are calculated from the measured gain and calculated directivity values at broadside. For example, for $V_{1}=0 \mathrm{~V}$ and $V_{2}=1 \mathrm{~V}$ the directivity of the antenna at the first and second band are, respectively, 1.76 and $1.96 \mathrm{dBi}$, which corresponds to an efficiency of $58 \%$ and $59 \%$ for the first and second bands, respectively. As the bias voltages increase, so do these efficiency values. For example, when $V_{1}=V_{2}=30 \mathrm{~V}$, the directivity of the antenna at the first and second bands are 1.82 and $2.16 \mathrm{dBi}$, respectively. This corresponds to efficiency values of $87 \%$ and $92 \%$ at the first and second bands, respectively.

\section{CONCLUSION}

A new technique for designing dual-band reconfigurable slot antennas is proposed. The technique is successfully applied to design a dual-band slot antenna with consistent radiation patterns at both bands and over the entire frequency range of the antenna. Measurement results of the antenna indicate that its frequency ratio can assume any value in the range of $1.3 \leq$ $f_{R} \leq 2.67$ by changing the dc bias voltages of its two varactors in the range of $0.5 \mathrm{~V} \leq V_{1}, V_{2} \leq 30 \mathrm{~V}$. Furthermore, good simultaneous matching is observed at both bands for the entire range of bias voltages, low levels of cross-polarized radiation is observed, and the radiation patterns of each band remain practically unchanged as the dc bias voltage is changed.

\section{REFERENCES}

[1] K. L. Wong, Compact and Broadband Microstrip Antennas. New York: Wiley, 2002.

[2] S. Maci, B. B. Gentili, P. Piazzesi, and C. Salvador, "Dual band slot-loaded patch antenna," Proc. Inst. Elect. Eng. Microw. Antennas Propag., vol. 142, no. 3, pp. 225-232, Jun. 1995.

[3] K. B. Hsieh and K. Wong, "Inset microstrip-line-fed dual-frequency circular microstrip antenna and its application to a two-element dualfrequency microstrip array," Proc. Inst. Elect. Eng. Microw. Antennas Propag., vol. 147, pp. 359-361, Oct. 1999.
[4] S. T. Fang and K. L. Wong, "A dual frequency equilateral triangular microstrip antenna with a pair of two narrow slots," Microw. Opt. Technol. Lett., vol. 23, pp. 82-84, Oct. 1999.

[5] D. Llorens, P. Otero, and C. C. Penalosa, "Dual-band, single CPW port, planar slot antenna," in Proc. IEEE Antennas and Propagation Society Int. Symp., vol. 51, Jan. 2003, pp. 137-139.

[6] C. Wang, J. Lee, and R. Huang, "Experimental studies of a miniaturized CPW-fed slot antenna with the dual-frequency operation," IEEE Antennas Wireless Propag. Lett., vol. 2, pp. 151-154, 2003.

[7] T. Morioka, S. Araki, and K. Hirasawa, "Slot antenna with parasitic element for dual band operation," Electron. Lett., vol. 33, pp. 2093-20944, Dec. 1997.

[8] J. S. Chen, "Triple-frequency annular-ring slot antennas fed by CPWand microstrip line," in Proc. IEEE APS/URSI Int. Symp., vol. 2, 2003, pp. 557-560.

[9] N. Behdad and K. Sarabandi, "A wide-band slot antenna design employing a fictitious short circuit concept," IEEE Trans. Antennas Propag., vol. 53, pp. 475-482, Jan. 2005.

[10] R. Azadegan and K. Sarabandi, "A novel approach for miniaturization of slot antennas," IEEE Trans. Antennas Propag., vol. 51, pp. 421-429, 2003.

[11] N. Behdad and K. Sarabandi, "Bandwidth enhancement and further size reduction of a class of miniaturized slot antennas," IEEE Trans. Antennas Propag., vol. 52, pp. 1928-1935, Aug. 2004.

[12] D. Peroulis, K. Sarabandi, and L. P. B. Katehi, "Design of reconfigurable slot antennas," IEEE Trans. Antennas Propag., vol. 53, pp. 645-654, Feb. 2005.

[13] J. T. Aberle, O. Sung-Hoon, D. T. Auckland, and S. D. Rogers, "Reconfigurable antennas for wireless devices," IEEE Antennas Propag. Mag., vol. 45, pp. 148-154, Dec. 2003.

[14] B. A. Cetiner, H. Jafarkhani, Q. Jiang-Yuan, Y. H. Jae, A. Grau, and F. De Flaviis, "Multifunctional reconfigurable MEMS integrated antennas for adaptive MIMO systems," IEEE Commun. Mag., vol. 42, pp. 62-70, Dec. 2004.

[15] P. J. Rainville and F. J. Harackiewicz, "Magnetic tuning of a microstrip patch antenna fabricated on a ferrite film," IEEE Microw. Guided Wave Lett., vol. 2, pp. 483-485, Dec. 1992.

[16] S. Zhang, G. H. Huff, J. Feng, and J. T. Bernhard, "A pattern reconfigurable microstrip parasitic array," IEEE Trans. Antennas Propag., vol. 52, pp. 2773-2776, Oct. 2004

[17] N. Behdad and K. Sarabandi, "A varactor tuned dual-band slot antenna," IEEE Trans. Antennas Propag., Special Issue on Multifunction Antennas and Antenna Systems, pt. II, vol. 54, no. 1, pp. 401-408, Jan. 2006.

[18] Garg et al., Microstrip Antenna Design Handbook. Norwood, MA Artech House, 2001, ch. 7.

[19] D. M. Pozar, Microwave Engineering. New York: Wiley, 2004.

[20] Gupta et al., Microstrip Lines and Slotlines, 2nd ed. Norwood, MA: Artech House, 1996.

[21] R. Janaswamy and D. H. Schaubert, "Characteristic impedance of a wide slotline on low-permittivity substrates," IEEE Trans. Microw. Theory Tech., vol. 34, no. 8, pp. 900-902, Aug. 1986.

[22] L. J. Chu, "Physical limitations on omni-directional antennas," J. Appl. Phys., vol. 19, pp. 1163-1175, Dec. 1948.

[23] J. P. Kim and W. S. Park, "Network modeling of an inclined and offcenter microstrip-fed slot antenna," IEEE Trans. Antennas Propag., vol. 46, pp. 1182-1188, 1998

[24] IE3D, Zeland Software Co. 


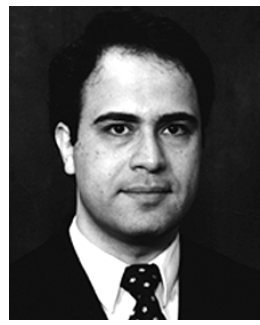

Nader Behdad (S'98) was born in Mashhad, Iran, in 1977. He received the Bachelor of Science degree in electrical engineering from Sharif University of Technology, Tehran, Iran, in September 2000 and the Master of Science degree in electrical engineering from the University of Michigan, Ann Arbor, in 2003. He is currently working toward the Ph.D. degree in the Department of Electrical Engineering and Computer Science, University of Michigan.

From 2000 to 2001, he worked as a Design Engineer for the Electronics Research Center, Sharif University of Technology. Since January 2002, he has been working as a Research Assistant at the Center for Wireless Integrated Micro-Systems, University of Michigan.

Mr. Behdad is the recipient of the Best Student Paper Award in the Antenna Applications Symposium held in Monticelo, IL, in September 2003, winner of the second prize in the student paper competition of the USNC/URSI National Radio Science Meeting, Boulder, CO, in January 2004, and the recipient of the Horace H. Rackham Predoctoral Fellowship from the Rackham School of Graduate Studies, University of Michigan in 2005.

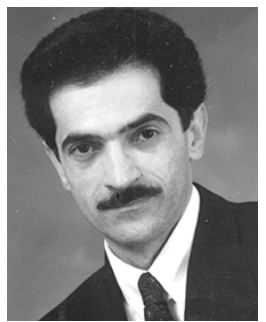

Kamal Sarabandi (S'87-M'90-SM'92-F'00) received the B.S. degree in electrical engineering from Sharif University of Technology, Tehran, Iran, in 1980, the M.S. degree in electrical engineering/mathematics, and the Ph.D. degree in electrical engineering from The University of Michigan-Ann Arbor, in 1986 and 1989, respectively.

$\mathrm{He}$ is the Director of the Radiation Laboratory and a Professor with the Department of Electrical Engineering and Computer Science, University of Michigan. His research areas of interest include microwave and millimeter-wave radar remote sensing, electromagnetic wave propagation, and antenna miniaturization. He has 20 years of experience with wave propagation in random media, communication channel modeling, microwave sensors, and radar systems, and is leading a large research group including two research scientists, 10 Ph.D., and two M.S. students. Over the past 10 years, he has graduated 21 Ph.D. students. He has served as the Principal Investigator on many projects sponsored by NASA, JPL, ARO, ONR, ARL, NSF, DARPA, and numerous industries. He has published many book chapters and more than 120 papers in refereed journals on electromagnetic scattering, random media modeling, wave propagation, antennas, meta-materials, microwave measurement techniques, radar calibration, inverse scattering problems, and microwave sensors. He has also had more than 270 papers and invited presentations in many national and international conferences and symposia on similar subjects.

Dr. Sarabandi is a vice president of the IEEE Geoscience and Remote Sensing Society (GRSS), and a member of IEEE Technical Activities Board Awards Committee. He is serving as the Associate Editor of the IEEE TRANSACTIONS on Antennas and Propagation and the IEEE Sensors Journal. He is also a member of Commissions F and D of URSI and of The Electromagnetic Academy. He is listed in American Men and Women of Science Who's Who in America and Who's Who in Science and Engineering. He was the recipient of the Henry Russel Award from the Regent of The University of Michigan (the highest honor the University of Michigan bestows on a faculty member at the assistant or associate level). In 1999, he received a GAAC Distinguished Lecturer Award from the German Federal Ministry for Education, Science, and Technology given to about 10 individuals worldwide in all areas of engineering, science, medicine, and law. He was also a recipient of a 1996 EECS Department Teaching Excellence Award and a 2004 College of Engineering Research Excellence Award. In 2005, he received two prestigious awards, namely, the IEEE Geoscience and Remote Sensing Distinguished Achievement Award and the University of Michigan Faculty Recognition Award. In the past several years, joint papers presented by his students at a number of international symposia (IEEE AP'95,'97,'00,'01,'03, IEEE IGARSS'99,'02, IEEE IMS'01, URSI' 04,'05) have received top student prize paper awards. 